\title{
The Herglotz principle and vakonomic dynamics
}

\author{
Manuel de León@*i \\ mdeleon@icmat.es \\ Manuel Lainz@] ${ }^{*}$ \\ manuel.lainz@icmat.es \\ Miguel C. Muñoz-Lecandaㅁำ \\ miguel.carlos.munoz@upc.edu
}

June 7, 2021

\begin{abstract}
In this paper we study vakonomic dynamics on contact systems with nonlinear constraints. In order to obtain the dynamics, we consider a space of admisible paths, which are the ones tangent to a given submanifold. Then, we find the critical points of the Herglotz action on this space of paths. This dynamics can be also obtained through an extended Lagrangian, including Lagrange multiplier terms.

This theory has important applications in optimal control theory for Herglotz control problems, in which the cost function is given implicitly, through an ODE, instead of by a definite integral. Indeed, these control problems can be considered as particular cases of vakonomic contact systems, and we can use the Lagrangian theory of contact systems in order to understand their symmetries and dynamics.
\end{abstract}

Keywords: Contact Hamiltonian systems, Constrained systems, Vakonomic dynamics, Optimal Control.

\section{Introduction}

Given a Lagrangian $L: T Q \rightarrow \mathbb{R}$ and a submanifold $N \subseteq T Q$, one can look for the critical points of the Euler-Lagrange action restricted to the paths which are tangent to $N$. This critical points are the solutions of the Euler-Lagrange equations for the extended Lagrangian [3] $\mathcal{L}\left(q^{i}, \dot{q}^{i}, \lambda_{a}\right)=L\left(q^{i}, \dot{q}^{i}\right)-\lambda_{a} \phi^{a}$, where $\left\{\phi^{a}\right\}$ are a set of independent

*Instituto de Ciencias Matemáticas (CSIC-UAM-UC3M-UCM), Madrid, Spain

${ }^{\dagger}$ Real Academia de Ciencias Exactas, Físicas y Naturales, Madrid, Spain

${ }^{\ddagger}$ Department of Mathematics, Universitat Politècnica de Catalunya 
constraints defining $N$. We remark that the dynamics obtained from this principle is, in general, different to nonholonomic dynamics [6, 13, in which the critical points of the action are computed on the unconstrained space of paths, but the admisible variations are constrained.

While nonholonomic dynamics has applications in engineering problems, vakonomic mechanics can be used to study optimal control problems. This opens up the possibility to apply results and techniques from Lagrangian mechanics to the study of optimal control problems, such as the Noether theorem and its generalizations [15], or variational integrators constructed from the theory of discrete mechanics [4].

On the other hand, in the Herglotz variational principle one considers a Lagrangian $L: T Q \times \mathbb{R} \rightarrow \mathbb{R}, L\left(q^{i}, \dot{q}^{i}, z\right)$ that depends not only on the positions and velocities of the system, but also on the action $z$ itself. The action is then defined implicitly, through the ODE $\dot{z}=L\left(q^{i}, \dot{q}^{i}, z\right)$. The critical points of this action are the solutions of the Herglotz equations [12, 14]:

$$
\frac{\partial L}{\partial q^{i}}-\frac{\mathrm{d}}{\mathrm{d} t} \frac{\partial L}{\partial \dot{q}^{i}}=\frac{\partial L}{\partial \dot{q}^{i}} \frac{\partial L}{\partial z} .
$$

It has recently been acknowledged that the Herglotz principle provides the dynamics for the Lagrangian counterpart of contact Hamiltonian systems [9]. This has allowed the developement of a theory of symmetries [10, 11] which in this setting are not related to conserved quantities, but to dissipated ones, which decay at the same rate as the energy. Furthermore variational integrators based on the Herglotz principle have been developed [19, 2].

Contact dynamics and the Herglotz principle have applications on the description of many physical systems, such as mechanical systems with friction, thermodynamic systems and some cosmological models [5, 16, 17, 18].

While the dynamics of contact systems with (linear) nonholonomic constraints [7] has been studied, a theory of contact vakonomic dynamics has not still been developed. This theory could be useful for the study of the Herglotz Optimal Control Problem, introduced in [8], in which the cost function is defined by an ODE, instead of an integral. This will allow a new way to obtain the dynamical equations (on 8, they were obtained rather indirectly, throught Pontryaguin maximum principle) and to apply some of the results of contact Lagrangian systems to this situation.

The paper is structured as follows. In Section 2 we review the Herglotz principle. Its traditional formulation, in which the action is defined implicitly, makes the implementation of the constraints difficult. We present and alternative in which the action is defined explicitly, but vakonomic constraints are present. This will make the addition of new constraints almost trivial. In Section 3 we obtain the vakonomic dynamical equations for a constrained contact system, and see that this dynamics can also be obtained through an extended contact Lagrangian. Finally, in Section 4, we sketch the relationship between the vakonomic dynamics of contact Lagrangian systems and the Herglotz optimal control problem. 


\subsection{The Herglotz variational principle, revisited}

Let $Q$ be the configuration manifold and let $L: T Q \times \mathbb{R} \rightarrow \mathbb{R}$ be the contact Lagrangian.

Consider the (infinite dimensional) manifold $\Omega\left(q_{0}, q_{1}\right)$ of curves $c:[0,1] \rightarrow Q$ with endpoints $q_{0}, q_{1} \in Q$. That is, $c(0)=q_{0}, c(1)=q_{1}$. The tangent space of $\Omega\left(q_{0}, q_{1}\right)$ at the curve $c$, is the space of vector fields along $c$ vanishing at the endpoints. That is,

$$
\begin{gathered}
T_{c} \Omega\left(q_{0}, q_{1}\right)=\left\{\delta c:[0,1] \rightarrow T Q \mid \delta c(t) \in T_{c(t)} Q \text { for all } \mathrm{t} \in[0,1],\right. \\
\delta c(0)=0, \delta c(1)=0\} .
\end{gathered}
$$

We fix a real number $z_{0} \in \mathbb{R}$ and consider the following operator:

$$
\mathcal{Z}: \Omega\left(q_{0}, q_{1}\right) \rightarrow \mathcal{C}^{\infty}([0,1] \rightarrow \mathbb{R}),
$$

which assigns to each curve $c$ the function $\mathcal{Z}(c)$ that solves the following ODE:

$$
\left\{\begin{array}{l}
\frac{\mathrm{d} \mathcal{Z}(c)}{\mathrm{d} t}=L(c, \dot{c}, \mathcal{Z}(c)), \\
\mathcal{Z}(c)(0)=z_{0},
\end{array}\right.
$$

that is, it assigns to each curve on the base space, its action as a function of time.

Now, the contact action functional maps each curve $c \in \Omega\left(q_{0}, q_{1}\right)$ to the increment of the solution of the ODE:

$$
\begin{aligned}
\mathcal{A}: \Omega\left(q_{0}, q_{1}\right) & \rightarrow \mathbb{R}, \\
c & \mapsto \mathcal{Z}(c)(1)-\mathcal{Z}(c)(0) .
\end{aligned}
$$

Note that, by the fundamental theorem of calculus,

$$
\mathcal{A}(c)=\int_{0}^{1} L(c(t), \dot{c}(t), \mathcal{Z}(c)(t)) \mathrm{d} t .
$$

Thus, in the case that $L$ does not depend on $z$, this coincides with the classical EulerLagrange action.

Remark 1. The Herglotz action is usually defined as $\mathcal{A}_{0}(c)=\mathcal{Z}(c)(1)$. However, this definition and our definition only differ by a constant. Indeed,

$$
\mathcal{A}(c)=\mathcal{A}_{0}(c)-z_{0} .
$$

In particular they have the same critical points. However the computations in the vakonomic principle are simpler for $\mathcal{A}$.

As it is proved in [9], the critical points of this action functional are precisely the solutions to Herglotz equation:

Theorem 1 (Herglotz variational principle). Let $L: T Q \times \mathbb{R} \rightarrow \mathbb{R}$ be a Lagrangian function and let $c \in \Omega\left(q_{0}, q_{1}\right)$ and $z_{0} \in \mathbb{R}$. Then, $(c, \dot{c}, \mathcal{Z}(c))$ satisfies the Herglotz equations:

$$
\frac{\mathrm{d}}{\mathrm{d} t} \frac{\partial L}{\partial \dot{q}^{i}}-\frac{\partial L}{\partial q^{i}}=\frac{\partial L}{\partial \dot{q}^{i}} \frac{\partial L}{\partial z},
$$

if and only if $c$ is a critical point of $\mathcal{A}$. 


\subsection{An alternative formulation of Herglotz variational principle}

Another way to approach this problem is to consider a constrained variational principle for curves on $Q \times \mathbb{R}$ constrained to a hypersurface $N$. We see that this is equivalent to the dynamics produced by a Lagrangian $L$ when considering unconstrained curves on $Q$.

We will work on the manifold $\bar{\Omega}\left(q_{0}, q_{1}, z_{0}\right)$ of curves $\bar{c}=\left(c, c_{z}\right):[0,1] \rightarrow Q \times \mathbb{R}$ such that $c(0)=q_{0}, c(1)=q_{1}, c_{z}(0)=z_{0}$. We do not constraint $c_{z}(1)$. The tangent space at the curve $c$ is given by

$$
\begin{aligned}
& T_{c} \bar{\Omega}\left(q_{0}, q_{1}, z_{0}\right)=\left\{\delta \bar{c}=\left(\delta c, \delta c_{z}\right):[0,1] \rightarrow T(Q \times \mathbb{R}) \mid\right. \\
& \left.\quad \delta \bar{c}(t) \in T_{c(t)}(Q \times \mathbb{R}) \text { for all } \mathrm{t} \in[0,1], \delta c(0)=0, \delta c(1)=0, \delta c_{z}(0)=0\right\} .
\end{aligned}
$$

In this space, the action functional $\overline{\mathcal{A}}$ can be defined as an integral

$$
\begin{aligned}
\overline{\mathcal{A}}: \bar{\Omega}\left(q_{0}, q_{1}, z_{0}\right) & \rightarrow \mathbb{R}, \\
\bar{c} & \mapsto z_{1}-z_{0}=\int_{0}^{1} \dot{c}_{z}(t) \mathrm{d} t .
\end{aligned}
$$

We will restrict this action to the set of paths that satisfy $\dot{c}_{z}=L$. For this, consider the hypersurface $N \subseteq T(Q \times \mathbb{R})$, which is the zero set of the constraint function $\phi$ :

$$
\phi(q, \dot{q}, z, \dot{z})=\dot{z}-L(q, \dot{q}, z) .
$$

Conversely, given any hypersurface $N$ transverse to the $\dot{z}$-parametric curves, by the implicit function theorem there exists locally a function $L$ such that $N$ is given by the equation $\dot{z}=L$. In this sense, we see that an hypersurface $N \subseteq T(Q \times \mathbb{R})$ is roughly equivalent to a Lagrangian $L: T Q \times \mathbb{R} \rightarrow \mathbb{R}$.

We consider the submanifold of curves tangent to $N$

$$
\bar{\Omega}_{N}\left(q_{0}, q_{1}, z_{0}\right)=\left\{\bar{c} \in \bar{\Omega}\left(q_{0}, q_{1}, z_{0}\right) \mid \dot{\bar{c}}(t) \in N \text { for all } \mathrm{t}\right\}
$$

Notice that the map $\operatorname{Id} \times \mathcal{Z}: \Omega\left(q_{0}, q_{1}\right) \rightarrow \bar{\Omega}_{N}\left(q_{0}, q_{1}, z_{0}\right)$ given by $(\operatorname{Id} \times \mathcal{Z})(c)=(c, \mathcal{Z}(c))$ is a bijection, with inverse $\operatorname{pr}_{Q}\left(c, c_{z}\right)=c$. Here, $\mathcal{Z}$, is defined on (3) . Moreover, the following diagram commutes

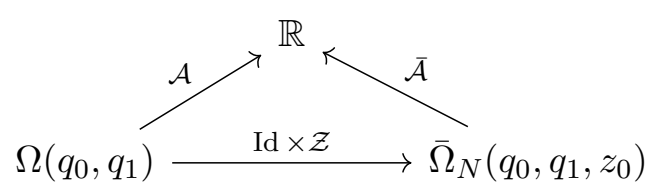

Hence $\bar{c} \in \bar{\Omega}_{N}\left(q_{0}, q_{1}, z_{0}\right)$ is a critical point of $\overline{\mathcal{A}}$ if and only if $c$ is a critical point of $\mathcal{A}$. So the critical points of $\mathcal{A}$ restricted to $\bar{\Omega}\left(q_{0}, q_{1}, z_{0}\right)$ are precisely the curves that satisfy the Herglotz equations.

We will also provide an alternate proof. We find directly the critical points of $\overline{\mathcal{A}}$ restricted to $\bar{\Omega}_{N}\left(q_{0}, q_{1}, z_{0}\right) \subseteq \bar{\Omega}\left(q_{0}, q_{1}, z_{0}\right)$ using the following infinite-dimensional version of the Lagrange multiplier theorem [1, 3.5.29]. 
Theorem 2 (Lagrange multiplier Theorem). Let $M$ be a smooth manifold and let $E$ be a Banach space such that $g: M \rightarrow E$ is a smooth submersion, so that $A=g^{-1}(\{0\})$ is a smooth submanifold. Let $f: M \rightarrow \mathbb{R}$ be a smooth function. Then $p \in A$ is a critical point of $\left.f\right|_{A}$ if and only if there exists $\hat{\lambda} \in E^{*}$ such that $p$ is a critical point of $f+\hat{\lambda} \circ g$.

We will apply this result to our situation. In the notation of this last theorem, $M=$ $\bar{\Omega}\left(q_{0}, q_{1}, z_{0}\right)$ is the smooth manifold. We pick the Banach space $E=L^{2}([0,1] \rightarrow \mathbb{R})$ of square integrable functions. This space is, indeed, a Hilbert space with inner product

$$
\langle\alpha, \beta\rangle=\int_{0}^{1} \alpha(t) \beta(t) \mathrm{d} t .
$$

We remind that, by the Riesz representation theorem, there is a bijection between $L^{2}([0,1] \rightarrow \mathbb{R})$ and its dual such that for each $\hat{\alpha} \in L^{2}([0,1] \rightarrow \mathbb{R})^{*}$ there exists $\alpha \in$ $L^{2}([0,1] \rightarrow \mathbb{R})$ with $\hat{\alpha}(\beta)=\langle\alpha, \beta\rangle$ for all $\beta \in L^{2}([0,1] \rightarrow \mathbb{R})$.

Our constraint function is

$$
\begin{aligned}
g: \bar{\Omega}\left(q_{0}, q_{1}, z_{0}\right) & \rightarrow L^{2}([0,1] \rightarrow \mathbb{R}), \\
\bar{c} & \mapsto(\phi) \circ(\bar{c}, \dot{\bar{c}}),
\end{aligned}
$$

where $\phi$ is a constraint locally defining $N$. Note that $A=g^{-1}(0)=\bar{\Omega}_{N}\left(q_{0}, q_{1}, z_{0}\right)$.

By Theorem 2, $c$ is a critical point of $f=\overline{\mathcal{A}}$ restricted to $\bar{\Omega}_{N}\left(q_{0}, q_{1}, z_{0}\right)$ if and only if there exists $\hat{\lambda} \in L^{2}([0,1] \rightarrow \mathbb{R})^{*}\left(\right.$ which is represented by $\left.\lambda \in L^{2}([0,1] \rightarrow \mathbb{R})\right)$ such that $c$ is a critical point of $\overline{\mathcal{A}}_{\lambda}=\overline{\mathcal{A}}+\hat{\lambda} \circ g$.

Indeed,

$$
\overline{\mathcal{A}}_{\lambda}=\int_{0}^{1} \mathcal{L}_{\lambda}(\bar{c}(t), \dot{\bar{c}}(t)) \mathrm{d} t
$$

where

$$
\mathcal{L}_{\lambda}(q, z, \dot{q}, \dot{z})=\dot{z}-\lambda \phi(q, z, \dot{q}, \dot{z}) .
$$

Since the endpoint of $c_{z}$ is not fixed, the critical points of this functional $\overline{\mathcal{A}}_{\lambda}$ are the solutions of the Euler-Lagrange equations for $\mathcal{L}_{\lambda}$ that satisfy the natural boundary condition:

$$
\frac{\partial \mathcal{L}_{\lambda}}{\partial \dot{z}}(\bar{c}(1), \dot{\bar{c}}(1))=1-\lambda(1) \frac{\partial \phi}{\partial \dot{z}}(\bar{c}(1), \dot{\bar{c}}(1))=0 .
$$

For $\phi=\dot{z}-L$, this condition reduces to $\lambda(1)=1$.

The Euler-Lagrange equations of $\mathcal{L}_{\lambda}$ are given by

$$
\begin{aligned}
& \frac{\mathrm{d}}{\mathrm{d} t}\left(\lambda(t) \frac{\partial \phi(\bar{c}(t), \dot{\bar{c}}(t))}{\partial \dot{q}^{i}}\right)-\lambda(t) \frac{\partial \phi(\bar{c}(t), \dot{\bar{c}}(t))}{\partial q^{i}}=0 \\
& \frac{\mathrm{d}}{\mathrm{d} t}\left(\lambda(t) \frac{\partial \phi(\bar{c}(t), \dot{\bar{c}}(t))}{\partial \dot{z}}\right)-\lambda(t) \frac{\partial \phi(\bar{c}(t), \dot{\bar{c}}(t))}{\partial z}=0
\end{aligned}
$$

since $\phi=\dot{z}-L$, the equation (18b) for $z$ is just

$$
\frac{\mathrm{d} \lambda(t)}{\mathrm{d} t}=-\lambda(t) \frac{\partial L}{\partial z}
$$

substituting on (18a) and dividing by $\lambda$, we obtain Herglotz equations. 


\subsection{Vakonomic constraints}

If we have more constraints, we can obtain vakonomic dynamics, just by changing $\phi$ by $\phi^{a}$ and $\lambda$ by $\lambda_{a}$ on (18), where $a$ ranges from 0 to the number of constraints $k$. Indeed, we restrict our path space to the ones tangent to submanifold $\tilde{N} \subseteq N \subseteq T(Q \times \mathbb{R})$, where $N$ is the zero set of $\phi^{0}$, given by $\phi^{0}=\dot{z}-L$. Repeating the similar computations, we would find that the critical points of $\left.\mathcal{A}\right|_{\Omega\left(q_{0}, q_{1}, \tilde{N}\right)}$ are the solutions of

$$
\begin{aligned}
\frac{\mathrm{d}}{\mathrm{d} t}\left(\lambda_{a}(t) \frac{\partial \phi^{a}(\bar{c}(t), \dot{\bar{c}}(t))}{\partial \dot{q}^{i}}\right)-\lambda_{a}(t) \frac{\partial \phi^{a}(\bar{c}(t), \dot{\bar{c}}(t))}{\partial q^{i}} & =0 \\
\frac{\mathrm{d}}{\mathrm{d} t}\left(\lambda_{a}(t) \frac{\partial \phi^{a}(\bar{c}(t), \dot{\bar{c}}(t))}{\partial \dot{z}}\right)-\lambda_{a}(t) \frac{\partial \phi^{a}(\bar{c}(t), \dot{\bar{c}}(t))}{\partial z} & =0, \\
\phi^{a}(\bar{c}(t), \dot{\bar{c}}(t)) & =0,
\end{aligned}
$$

where $\left(\phi^{a}\right)_{a=0}^{k}$ are constraints defining $\tilde{N}$ as a submanifold of $T Q \times \mathbb{R}$. Since $\frac{\partial \phi^{0}}{\partial \dot{z}}=0$, the rest of the constraints can be chosen to be independent of $\dot{z}$. We denote

$$
\begin{aligned}
\psi^{\alpha}(q, \dot{q}, z) & =\phi^{\alpha}(q, \dot{q}, z, L(q, \dot{q}, z)), \\
\mu_{\alpha} & =\frac{\lambda_{\alpha}}{\lambda_{0}} \\
\mathcal{L}_{\mu}(q, \dot{q}, z, t) & =L(q, \dot{q}, z)-\mu_{\alpha}(t) \psi^{\alpha}(q, \dot{q}, z)
\end{aligned}
$$

for $\alpha \in\{1, \ldots k\}$, provided that $\lambda_{0} \neq 0$.

From this, we can write the equations (20) as

$$
\begin{aligned}
-\frac{\mathrm{d}}{\mathrm{d} t}\left(\lambda_{0}(t) \frac{\partial \mathcal{L}_{\mu}}{\partial \dot{q}^{i}}\right)+\lambda_{0}(t) \frac{\partial \mathcal{L}_{\mu}}{\partial q^{i}} & =0 \\
\frac{\mathrm{d} \lambda_{0}(t)}{\mathrm{d} t}=\lambda_{0}(t) \frac{\partial \mathcal{L}_{\mu}}{\partial z} & =0, \\
\psi^{\alpha}(\bar{c}(t), \dot{c}(t)) & =0, \\
\dot{c}_{z}(t) & =\mathcal{L}_{\mu}(\bar{c}(t), \dot{c}(t), t) .
\end{aligned}
$$

Substituting (24b) onto (24a), dividing by $\lambda_{0}$ and reordering terms, we obtain

$$
\begin{aligned}
\frac{\mathrm{d}}{\mathrm{d} t}\left(\frac{\partial \mathcal{L}_{\mu}}{\partial \dot{q}^{i}}\right)-\frac{\partial \mathcal{L}_{\mu}}{\partial q^{i}} & =\frac{\partial \mathcal{L}_{\mu}}{\partial \dot{q}^{i}} \frac{\partial \mathcal{L}_{\mu}}{\partial z} \\
\psi^{\alpha}(\bar{c}(t), \dot{c}(t)) & =0 \\
\dot{c_{z}}(t) & =\mathcal{L}_{\mu}(\bar{c}(t), \dot{c}(t), t) .
\end{aligned}
$$

We remark that these equations are just the Herglotz equations for the extended Lagrangian $\mathcal{L}$ :

$$
\begin{aligned}
\mathcal{L}: T\left(Q \times \mathbb{R}^{k}\right) \times \mathbb{R} & \rightarrow \mathbb{R} \\
(q, \mu, \dot{q}, \dot{\mu}, z) & \mapsto L(q, \dot{q}, z)-\mu_{\alpha} \psi^{\alpha}(q, \dot{q}, z)
\end{aligned}
$$




\subsection{Applications to control}

The Herglotz optimal control problem [8] can be formulated by working on the control bundle $W \times \mathbb{R} \rightarrow Q \times \mathbb{R}$, with local coordinates $\left(x^{i}, u^{a}, z\right)$ : the variables $x^{i}$, the controls $u^{a}$ and the action $z$.

The problem consists on finding the curves $\gamma: I=[a, b] \rightarrow W, \gamma=\left(\gamma_{Q}, \gamma_{U}, \gamma_{z}\right)$, such that

1) end points conditions: $\gamma_{Q}(a)=x_{a}, \gamma_{Q}(b)=x_{b}, \gamma_{z}(a)=z_{0}$,

2) $\gamma_{Q}$ is an integral curve of $X: \dot{\gamma}_{Q}=X \circ\left(\gamma_{Q}, \gamma_{U}\right)$,

3) $\gamma_{z}$ satifies the differential equation $\dot{z}=F(x, u, z)$, and

4) maximal condition: $\gamma_{z}(b)$ is maximum over all curves satisfying 1$\left.)-3\right)$.

We remark that this can be interpreted as a vakonomic Herglotz principle on $T W$, with constraints given by the control equations $\phi^{i}=X^{i}(x, u, z)-\dot{q}^{i}$ and the Lagrangian being the cost function $L\left(x^{i}, u^{a}, \dot{q}^{i}, \dot{u}^{a}, z\right)=F(x, u, z)$. The equations of motion obtained through the contact vakonomic principle coincides with the ones obtained indirectly through Pontryaguin maximum principle in [8, Eq. 28]

$$
\begin{aligned}
\dot{q}^{i} & =X^{i}, \\
\dot{\mu}_{i} & =\frac{\partial F}{\partial x^{i}}-\mu_{j} \frac{\partial X^{j}}{\partial x^{i}}-\mu_{j}\left(\frac{\partial F}{\partial z}-\mu_{i} \frac{\partial X^{j}}{\partial z}\right) \\
& =\mu_{i} \frac{\partial F}{\partial z}-\mu_{j} \frac{\partial X^{j}}{\partial x^{i}}+\frac{\partial F}{\partial x^{i}}-\frac{\partial X^{j}}{\partial z} \mu_{i} \mu_{j}, \\
\dot{z} & =F
\end{aligned}
$$

subjected to the constraints

$$
\frac{\partial F}{\partial u^{a}}-\mu_{j} \frac{\partial X^{j}}{\partial u^{a}}=0
$$

\section{Acknowledgements}

M. de León and M. Lainz acknowledge the partial finantial support from MINECO Grants MTM2016- 76-072-P and the ICMAT Severo Ochoa project SEV-2015-0554. M. Lainz wishes to thank MICINN and ICMAT for a FPI-Severo Ochoa predoctoral contract PRE2018-083203. M.C. Muñoz-Lecanda acknowledges the financial support from the Spanish Ministerio de Ciencia, Innovación y Universidades project PGC2018-098265B-C33 and the Secretary of University and Research of the Ministry of Business and Knowledge of the Catalan Government project 2017-SGR-932. 


\section{References}

[1] Ralph Abraham, J. E. Marsden, and Tudor Ratiu, Manifolds, Tensor Analysis, and Applications, second ed., Applied Mathematical Sciences, Springer-Verlag, New York, 1988 (en).

[2] Alexandre Anahory Simoes, David Martín de Diego, Manuel Lainz Valcázar, and Manuel de León, On the Geometry of Discrete Contact Mechanics, Journal of Nonlinear Science 31 (2021), no. 3, 53 (en).

[3] V. I. Arnold, Mathematical methods of classical mechanics, 2nd ed ed., Graduate Texts in Mathematics, no. 60, Springer, New York, 1997 (eng).

[4] Roberto Benito and David Martín de Diego, Discrete vakonomic mechanics, Journal of Mathematical Physics 46 (2005), no. 8, 083521.

[5] Alessandro Bravetti, Contact Hamiltonian Dynamics: The Concept and Its Use, Entropy 19 (2017), no. 12, 535 (en).

[6] Jorge Cortés, Manuel de León, D Martín de Diego, and Sonia Martínez, Geometric description of vakonomic and nonholonomic dynamics. Comparison of solutions, SIAM Journal on Control and Optimization 41 (2002), no. 5, 1389-1412.

[7] Manuel de León, Víctor Manuel Jiménez, and Manuel Lainz Valcázar, Contact Hamiltonian and Lagrangian systems with nonholonomic constraints, Journal of Geometric Mechanics (2020).

[8] Manuel de León, Manuel Lainz, and Miguel C. Muñoz-Lecanda, Optimal control, contact dynamics and Herglotz variational problem, arXiv:2006.14326 [math-ph] (2020).

[9] Manuel de León and Manuel Lainz Valcázar, Singular Lagrangians and precontact Hamiltonian systems, International Journal of Geometric Methods in Modern Physics 16 (2019), no. 10, 1950158.

[10] _ Infinitesimal symmetries in contact Hamiltonian systems, Journal of Geometry and Physics 153 (2020), 103651 (en).

[11] Jordi Gaset, Xavier Gràcia, Miguel C. Muñoz-Lecanda, Xavier Rivas, and Narciso Román-Roy, New contributions to the Hamiltonian and Lagrangian contact formalisms for dissipative mechanical systems and their symmetries, International Journal of Geometric Methods in Modern Physics (2020).

[12] Bogdana Georgieva, Ronald Guenther, and Theodore Bodurov, Generalized variational principle of Herglotz for several independent variables. First Noether-type theorem, Journal of Mathematical Physics 44 (2003), no. 9, 3911-3927. 
[13] Xavier Gracia, Jesus Marin-Solano, and Miguel-C Munoz-Lecanda, Some geometric aspects of variational calculus in constrained systems, Reports on Mathematical Physics 51 (2003), no. 1, 127-148.

[14] Gustav Herglotz, Beruhrungstransformationen, Lectures at the University of Gottingen (Gottingen), 1930.

[15] Sonia Martínez, Jorge Cortés, and Manuel De León, Symmetries in vakonomic dynamics: Applications to optimal control, Journal of Geometry and Physics 38 (2001), no. $3-4,343-365$.

[16] Ryszard Mrugala, James D. Nulton, J. Christian Schön, and Peter Salamon, Contact structure in thermodynamic theory, Reports on Mathematical Physics 29 (1991), no. $1,109-121$.

[17] David Sloan, A new action for cosmology, arXiv preprint arXiv:2010.07329 (2020).

[18] Michael Usher, Local rigidity, contact homeomorphisms, and conformal factors, arXiv preprint arXiv:2001.08729 (2020).

[19] Mats Vermeeren, Alessandro Bravetti, and Marcello Seri, Contact variational integrators, Journal of Physics A: Mathematical and Theoretical 52 (2019), no. 44, 445206 (en). 